\title{
A Two-Level Detection Algorithm for Optical Fiber Vibration
}

\author{
Fukun BI, Xuecong REN*, Hongquan QU, and Ruiqing JIANG \\ College of Information Engineering, North China University of Technology, Beijing, 100144, China \\ *Corresponding author: Xuecong RENＥ-mail: 2013311020122@mail.ncut.edu.cn
}

\begin{abstract}
Optical fiber vibration is detected by the coherent optical time domain reflection technique. In addition to the vibration signals, the reflected signals include clutters and noises, which lead to a high false alarm rate. The "cell averaging" constant false alarm rate algorithm has a high computing speed, but its detection performance will be declined in nonhomogeneous environments such as multiple targets. The "order statistics" constant false alarm rate algorithm has a distinct advantage in multiple target environments, but it has a lower computing speed. An intelligent two-level detection algorithm is presented based on "cell averaging" constant false alarm rate and "order statistics" constant false alarm rate which work in serial way, and the detection speed of "cell averaging" constant false alarm rate and performance of "order statistics" constant false alarm rate are conserved, respectively. Through the adaptive selection, the "cell averaging" is applied in homogeneous environments, and the two-level detection algorithm is employed in nonhomogeneous environments. Our Monte Carlo simulation results demonstrate that considering different signal noise ratios, the proposed algorithm gives better detection probability than that of "order statistics".
\end{abstract}

Keywords: Optical fiber vibration, the adaptive selection, Monte Carlo simulation, two-level detection

Citation: Fukun BI, Xuecong REN, Hongquan QU, and Ruiqing JIANG, "A Two-Level Detection Algorithm for Optical Fiber Vibration," Photonic Sensors, 2015, 5(3): 284-288.

\section{Introduction}

Optical fiber vibration can be detected by the coherent optical time domain reflection technique which employs coherent detection [1], and the weak backscattering signal can be extracted effectively. Besides vibration, the reflected signals include clutters and noises, which lead to a high false alarm rate. Constant false alarm rate (CFAR) detection involves the estimation of the parameters of the local clutter and the setting of a threshold for decision so that a constant false alarm probability $\left(P_{f a}\right)$ is guaranteed for all values of unknown clutter parameters. A large number of CFAR detectors have been proposed with different local statistics of the background clutter [2-4]. The "cell averaging" (CA) [5] CFAR is the optimum CFAR algorithm in a homogeneous background, compared with CA-CFAR, the "order statistics" (OS) [6] CFAR processor has some detection wastage in homogenous environments, but has a distinct advantage in multiple target environments. However, the real-time performance cannot be guaranteed, because of taking a long time to rank.

Considering the development of the CFAR detector, there are plenty of researches trying to combine them so as to utilize the advantages of different methods and balance the detection algorithm performance in the homogeneous backgrounds and nonhomogeneous environments. 
"Mean of order statistics and cell averaging" (MOSCA) has been proposed in [7], ordered statistics (OS)-cell-averaging (CA)-greatest-of selection (GO) formed OSCAGO was presented in $[8,9]$, and ordered statistics (OS)-cell-averaging (CA)-smallest-of selection (SO) formed OSCASO was proposed in [9]. The key of these methods is that they use OS parametric estimation in the left sliding window and CA estimation separately in the right sliding window, then use the sum of estimation of the leading and lagging sliding window as the estimate of the total power level. By using the combined algorithm, the time for sorting samples is only the half of OS. Based on variability index (VI) CFAR [10], the modified algorithm was proposed in [11-13]. These methods utilize a background estimation algorithm which is a composite of the CA-CFAR, SO-CFAR, and GO-CFAR approaches, and take advantage of the excellent homogeneous environment performance. However, these algorithms are complex and have a low efficiency. The above detection algorithms need to be detected only once, which cannot balance multiple problems. For these problems that CA-CFAR exhibits severe performance degradation in the presence of multiple targets and the OS-CFAR costs long time for sorting, an adaptive CFAR is firstly proposed in this paper. In this algorithm, the homogeneity of background is estimated before detection. When the background is judged as homogeneous, CA-CFAR is applied. On the other hand, CA-OS-CFAR is used. For the CA-OS, the first level detection is CA-CFAR with a two-dimensional column window to improve the detecting speed and reduce the data to the next level detection. The second level detection, OS-CFAR, should be used to detect the outcome. In this method, the detection speed of CA-CFAR and performance of OS-CFAR are conserved, respectively. Finally, the performance is analyzed by Monte Carlo simulation, so that the feasibility and the availability can be proved.

\section{Principle of adaptive selection detection}

The CA-CFAR processor sets the threshold by using reference windows (also called as "sliding windows" in [14]) to estimate local statistics of the background. In this paper, a two-dimensional column window proposed in [15] is employed. The OS-CAFR processor sets the threshold by the $k$ th ordered value after ranking according to the increasing magnitude. The detection schematic is shown in Fig. 1.

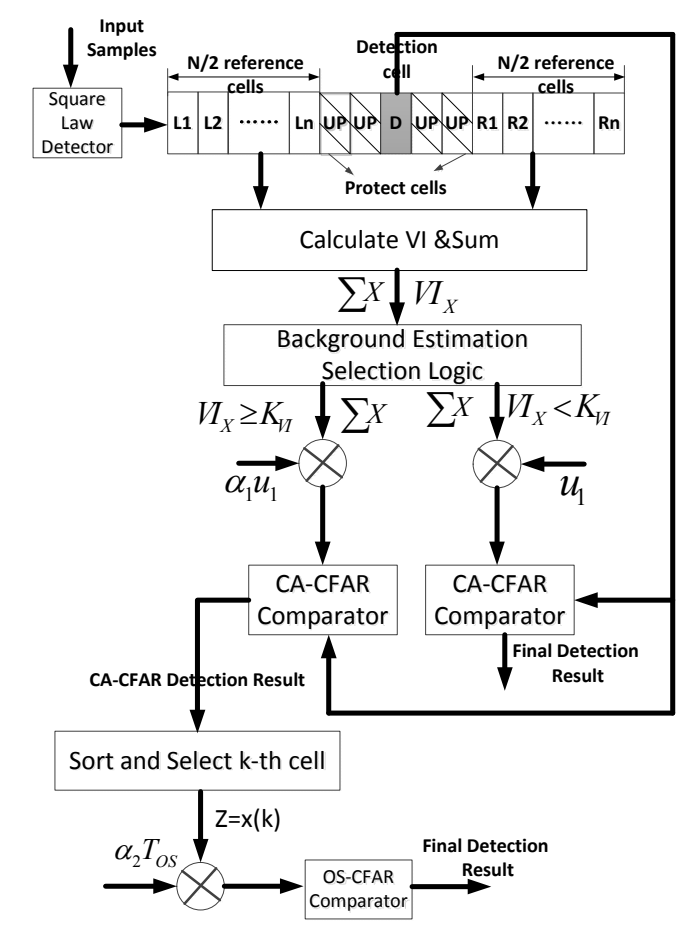

Fig. 1 Schematics of adaptive detection.

For the background estimation selection logic, the VI-CFAR's [10] method is employed. For implementation purposes, the simplified statistic $V I$ is employed as follows:

$$
\begin{aligned}
& V I=1+\frac{\sigma^{2}}{\mu}=1+\frac{1}{N} \sum_{i=1}^{N} \frac{\left(X_{i}-\bar{X}\right)^{2}}{\bar{X}^{2}} \\
& =\frac{\frac{1}{N} \sum_{i=1}^{N} X_{i}^{2}}{\left(\frac{1}{N} \sum_{i=1}^{N} X_{i}\right)^{2}}=\frac{N \sum_{i=1}^{N} X_{i}^{2}}{\left(\sum_{i=1}^{N} X_{i}\right)^{2}}
\end{aligned}
$$

where $\mu$ is the estimated population mean, $\sigma^{2}$ is the estimated population variance, and $\bar{X}$ is the 
arithmetic mean of the $N$ cells in a reference window.

The $V I$ is compared with a threshold $K_{V I}$ to decide if the cells with which the $V I$ is computed are from a homogeneous environment or from a nonhomogeneous environment using the following hypothesis test:

$$
\begin{gathered}
V I<K_{V I} \Rightarrow \text { Homogeneous } \\
V I \geq K_{V I} \Rightarrow \text { Nonhomogeneous } .
\end{gathered}
$$

For the hypothesis test, $\alpha_{0}$, given in (2), is defined as the probability of error such that a homogeneous environment is classified as variable

$$
\alpha_{0}=P\left[V I>K_{V I} \mid \text { Homogeneous Env. }\right] \text {. }
$$

By analyzing the detected signals, the in-phase and quadrature signals are independent, identically distributed (IID), Gaussian random processes. Consequently, the envelope amplitude at the output of a square-law detector is an exponentially distributed random variable. Based on the formula (1), through Monte Carlo simulation, if the $\alpha_{0}$ and $N$ are given, then $K_{V I}$ can be calculated as shown in Table 1.

Table1 $K_{V I}$ for background estimation selection logic.

\begin{tabular}{ccccc}
\hline$\alpha_{0}$ & $N=10$ & $N=20$ & $N=30$ & $N=40$ \\
\hline 0.1 & 2.5212 & 2.4401 & 2.3864 & 2.3497 \\
0.05 & 2.8296 & 2.6750 & 2.5796 & 2.5145 \\
0.01 & 3.5768 & 3.2608 & 3.0510 & 2.9154 \\
0.005 & 3.9389 & 3.5340 & 3.2720 & 3.0972 \\
0.001 & 4.7269 & 4.2051 & 3.8281 & 3.5765 \\
0.0005 & 5.0994 & 4.5611 & 4.0887 & 3.7754 \\
0.0001 & 5.9150 & 5.3367 & 4.6973 & 4.3595 \\
\hline
\end{tabular}

For CA-CFAR, according to [16], it can be known that

$$
T_{1}=P_{f a}^{-\frac{1}{N}}-1
$$

where $P_{f a}$ is the probability of false alarm, $N$ is the length of the reference window, and $T$ is the scale factor. While detecting by CA-CFAR, the number of reference cells is $N=2 c \times d$, that is the product of the length of reference cells $2 c$ and the length of time dimension $d$ with using the 2D column window. The threshold coefficient of the first level detection is shown as follows:

$$
u_{1}=2 c \times d \times\left(P_{f a}^{-\frac{1}{2 c d}}-1\right) .
$$

For OS-CFAR, the probability of false alarm is given by [5]

$$
\begin{gathered}
P_{f a}=\left(\frac{N}{k}\right) \frac{k !\left(u_{2}+N-k\right)}{\left(u_{2}+N\right) !}= \\
\left(\frac{N}{k}\right) \frac{\Gamma(k+1) \Gamma\left(u_{2}+N+1-k\right)}{\Gamma\left(u_{2}+N+1\right)} .
\end{gathered}
$$

According to the increasing magnitude, the amplitude values taken from the reference window are rank-ordered, and the $k$ th ordered value is selected as the mean level statistic estimation. For a $P_{f a}$, the threshold coefficient can be computed iteratively from (5) while the reference window size $N$ and the order number $k$ already have been given.

By CA-CFAR detection, the probability density function of the data is as follows:

$$
\begin{aligned}
& f_{\hat{y}}(\hat{y})=\int_{u_{1} \hat{y}}^{\infty} \frac{1}{2 \sigma^{2}} \exp \left(-\frac{y}{2 \sigma^{2}}\right) d y \times \\
& \frac{1}{2^{N} \Gamma(N)}\left(\frac{N}{\sigma^{2}}\right)^{N} \hat{y}^{N-1} \exp \left(-\frac{N \hat{y}}{2 \sigma^{2}}\right) .
\end{aligned}
$$

For the resultant probability of false alarm, it can be calculated by the following equation:

$$
P_{f a}=\int_{0}^{\infty} f_{Z}(z) \int_{u_{2} z}^{\infty} f_{\hat{y}}(\hat{y}) d \hat{y} d z
$$

where, based on [16], $f_{Z}(z)$ is defined as follows:

$$
f_{Z}(z)=\frac{k}{\mu}\left(\frac{N}{k}\right) e^{-(N-k+1) z / \mu}\left(1-e^{-z / \mu}\right)^{k-1} .
$$

Therefore, the resultant probability of false alarm is as follows:

$$
P_{f a}=\int_{0}^{\infty} f_{Z}(z) \int_{u_{2} z}^{\infty} f_{\hat{y}}(\hat{y}) d \hat{y} d z=
$$

$\frac{1}{2^{N}(N-1) !}\left(\frac{N}{\sigma^{2}}\right)^{N} \int_{0}^{\infty} f_{Z}(z) \int_{u_{2} z}^{\infty} \hat{y}^{N-1} \exp \left(-\frac{u_{1}+N}{2 \sigma^{2}} \hat{y}\right) d \hat{y} d z$.

Because it is difficult to analyze the relationship between two thresholds and the resultant probability of false alarm, so the numerical approximation by the Monte-Carlo experiment is employed.

Only the data crossed both the first level detection threshold given by CA-CFAR and the 
second level detection threshold given by OS-CFAR can be detected by using the two-level CFAR detection algorithm. Therefore, the practical false alarm rate is lower than the set false alarm rate in this way. Because the analytical method is difficult to implement, so Monte-Carlo simulation is employed, and the first threshold coefficients $u_{1}$ and the second threshold coefficients $u_{2}$ should be multiplied by coefficients $\alpha_{1}$ and $\alpha_{2}$ so as to achieve the set false alarm rate, respectively. In this paper, the CA-CFAR, OS-CFAR, and CA-OS-CFAR are proposed to detect the same data file, respectively. The false alarm time is given that $T_{f a}=5 \mathrm{~min}$, based on the given relationship between the false alarm rate and time by (10):

$$
P_{f a}=\frac{\tau}{T_{f a}} .
$$

In our system, the pulse width is $\tau=16 \mathrm{~ms}$, and a given $P_{f a}$ is $16 /(5 \times 60 \times 1000)=5.33 \times 10^{-5}$. The Monte-Carlo trails need to be implemented by 10000 times to choose the value of $\alpha_{1}$ and $\alpha_{2}$. From the trails, when $\alpha_{1}=0.9$ and $\alpha_{2}=0.9$, the three false alarm rates are shown in Table 2, respectively.

Table $2 P_{f a}$ comparison for three kinds of CFAR in homogeneous environments.

\begin{tabular}{cc}
\hline CFAR & $P_{f a}$ \\
\hline CA & $5.3526^{*} 10^{-5}$ \\
OS & $5.3105 * 10^{-5}$ \\
CA-OS & $5.3259 * 10^{-5}$ \\
\hline
\end{tabular}

From Table 2, the actual false alarm rate is close to the set value, therefore, the two coefficients are both chosen as 0.9 .

For the first level detection, the arithmetic speed increases by using a 2D slip window, then the least amount of data is detected by the second level detection. After two-level detection, the interference of the interfering targets decreases, and the detection speed of CA-CFAR and performance of OS-CFAR are conserved, respectively. As shown in Table 2, the same data are carried out by OS-CFAR, CA-CFAR, and CA-OS-CFAR, respectively, and the time taken by the experiments is shown in Table 3 .
Table 3 Execution time comparison for three kinds of CFAR detection algorithm.

\begin{tabular}{cccccc}
\hline & $N$ & 20 & 30 & 40 & 50 \\
\hline CAA & 4.25786 & 4.01248 & 4.14747 & 4.16042 & 4.23280 \\
CA-OS & 4.54096 & 4.40214 & 4.42973 & 4.50570 & 4.47693 \\
OS & 11.8978 & 11.5991 & 11.6829 & 11.9536 & 11.8496 \\
\hline
\end{tabular}

From Table 3, it can be known that the speed of CA-CFAR with the two-dimensional column window is the fastest, and the speed of OS-CFAR is the slowest. Compared with OS-CFAR, the speed is fast about three times, and it is faster than that of the algorithm of combined by CA and OS proposed in [7-9].

\section{Analysis of performance}

The detection performance of the presented detection algorithm and OS-CFAR algorithm are analyzed by using Monte-Carlo simulation. The length of reference cell is $N=40$, and the given false alarm rate is $P_{f a}=5.33 * 10^{-5}$, For the OS-CFAR, CA-OS-CFAR, and the presented algorithm, the order number $k=30$. The performance curves are given in Fig. 2 while only one target exists in the reference window.

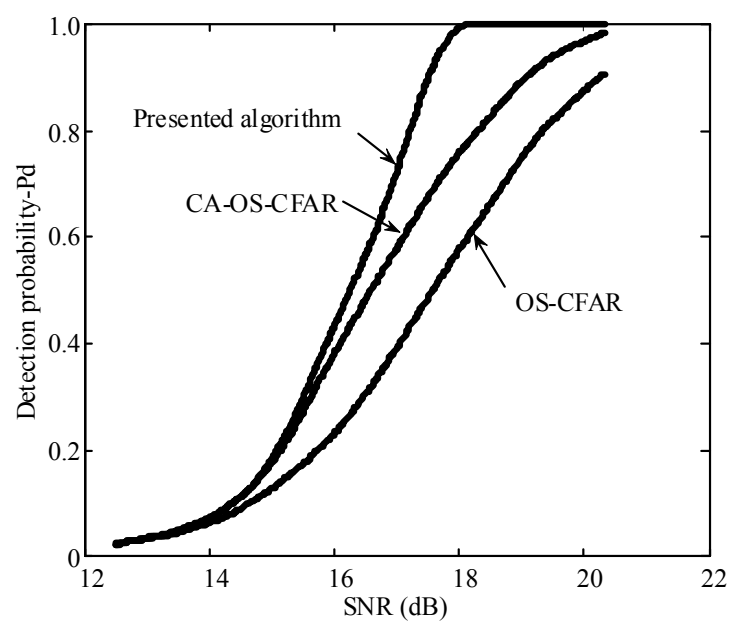

Fig. 2 Detection probability comparison of OS-CFAR and CA-OS-CFAR in different signal noise ratios (SNRs).

From the performance curves shown in Fig. 2, the performance of the presented detection algorithm is much better than that of CA-OS and OS-CFAR while one target exists in the reference window. 


\section{Conclusions}

For signals detected by the COTDR technique, a research by using the CFAR detection algorithm to decrease the false alarm rate of detection signals is presented. The CA-CFAR algorithm exhibits severe performance degradation in the presence of multiple targets, and the OS-CFAR algorithm costs long time for sorting. To overcome these problems, an adaptive CFAR algorithm based on ordered data variability has been proposed. It does not require any prior information about the environments, which is a choice between CA-CFAR and the two-level CFAR detection algorithm including CA-CFAR and OS-CFAR which work in serial way, and the detection speed of CA-CFAR and performance of OS-CFAR are conserved, respectively. In this algorithm, the relationship of two threshold coefficients is determined by Monte-Carlo simulation, and the relative formulas haven't been derived. Further research will be needed to derive the detailed formulas for calculating threshold coefficients.

\section{Acknowledgment}

This work is supported by "Scientific pre-research foundation of North China University of Technology" and "General project of science and technology program of Beijing Education Commission".

Open Access This article is distributed under the terms of the Creative Commons Attribution License which permits any use, distribution, and reproduction in any medium, provided the original author(s) and source are credited.

\section{References}

[1] X. Li, H. Liang, W. Xu, and X. Zhang, "Comparison of characteristics of commonly-used distributed optical fiber sensors," Optical Communication Technology, 2007, 31(5): 14-18.

[2] D. J. Crisp, The state-of-the-art in ship detection in synthetic aperture radar imagery. Australia: Defence Science and Technology Organisation, 2004.

[3] A. Pourmottaghi, M. R. Taban, and S. A. Gazor, "CFAR detector in a nonhomogenous Weibull clutter," IEEE Transactions on Aerospace and Electronic Systems, 2012, 48(2): 1747-1758.

[4] J. Liu, Z. Zhang, Y. Yang, and H. Liu, "A CFAR adaptive subspace detector for first-order or second-order Gaussian signals based on a single observation," IEEE Transactions on Signal Processing, 2011, 59(11): 5126-5140.

[5] H. M. Finn and R. S. Johnson, "Adaptive detection mode with threshold control as a function of spatially sampled clutter level estimates," RCA Review, 1968, 29(3): 414-464.

[6] H. Rohling, "Radar CFAR thresholding in clutter and multiple target situations," IEEE Transactions on Aerospace and Electronic Systems, 1983, 19(3): 608-621.

[7] Y. He, J. Guan, Y. Peng, and D. Lu, "A new CFAR detector based on order statistic and cell averaging," in Conference on CIE 1996 International Radar, Beijing, pp. 106-108, 1996.

[8] Y. He and J. Guan, "A new CFAR detector with greatest of selection," in Conference on Radar IEEE 1995 International, Alexandria, USA, pp. 589-591, 1995.

[9] Y. He and J. Guan, "Two new CFAR detectors based on greatest of and smallest of selection," Systems Engineering and Electronics, 1995, 17(7): 6-16.

[10] M. E. Smith and P. K. Varshney, "Intelligent CFAR processor based on data variability," IEEE Transactions on Aerospace and Electronic Systems, 2000, 36(3): 837-847.

[11] C. Hao, C. Hou, and W. Wang, "Distributed CFAR detection based on modified VI-CFAR algorithm," Journal of System Simulation, 2007, 19(4): 830-832.

[12] C. Xu, T. Jian, Y. He, and X. Gu, "An improved VI-CFAR detector," Signal Processing, 2011, 27(6): 926-931.

[13] H. Yu, J. Tao, and L. An, "VI-CFAR detector based on censored mean level," Modern Defence Technology, 2013, 41(4): 135-140.

[14] G. Gao, L. Liu, L. Zhao, G. Shi, and G. Kuang, "An adaptive and fast CFAR algorithm based on automatic censoring for target detection in high-resolution SAR images," IEEE Transactions on Geoscience and Remote Sensing, 2009, 47(6): 1685-1697.

[15] Q. Pan and F. Li, "Fast algorithm of target detection for terminal guidance radar," Guidance \& Fuze, 2007, 28(2): 16-19.

[16] P. P. Gandhi and S. A. Kassam, "Analysis of CFAR processors in nonhomogeneous background," IEEE Transactions on Aerospace and Electronic Systems, 1988, 24(4): 427-445. 